\title{
QUATERNIONIC PLURISUBHARMONIC FUNCTIONS AND THEIR APPLICATIONS TO CONVEXITY
}

\author{
S. ALESKER
}

Dedicated to Professor Victor Abramovich Zalgaller on the occasion of his 85th birthday

\begin{abstract}
The paper is a survey of the recent theory of plurisubharmonic functions of quaternionic variables, together with its applications to the theory of valuations on convex sets and HKT-geometry (Hyper-Kähler with Torsion). The exposition follows some earlier papers by the author and a joint paper by Verbitsky and the author.
\end{abstract}

\section{$\S 0$. INTRODUCTION}

Our goal in this article is to present a survey of the recent theory of plurisubharmonic functions of quaternionic variables, together with its applications to the theory of valuations on convex sets and HKT-geometry (Hyper-Kähler with Torsion). The exposition follows the papers $[4,5,7]$ by the author and [8] by Verbitsky and the author.

We denote by $\mathbb{H}$ the (noncommutative) field of quaternions. The notion of a quaternionic plurisubharmonic function on the flat space $\mathbb{H}^{n}$ was introduced by the author in [4] and independently by Henkin in [24]. This notion is a quaternionic analog of a convex function on $\mathbb{R}^{n}$ and a complex plurisubharmonic function on $\mathbb{C}^{n}$; see Definition 3.1 below. On the one hand, this class of functions enjoys many analytic properties similar to those of convex and complex plurisubharmonic functions. On the other hand, these properties reflect rather different geometric structures behind them. This will be illustrated below by applications to convexity and HKT-geometry.

We start with some analytic properties of quaternionic plurisubharmonic functions. In [4], the author proved a quaternionic analog of the Aleksandrov [2] and Chern-LevineNirenberg [19] theorems (see Theorems 3.4 and 3.6 in $\S 3$ below). It is worthwhile to recall these classical results now. The Aleksandrov theorem says that if a sequence $\left\{f_{N}\right\}$ of convex functions converges uniformly on compact subsets to a function $f$, then $f$ is convex, and

$$
\operatorname{det}\left(\frac{\partial^{2} f_{N}}{\partial x_{i} \partial x_{j}}\right) \stackrel{w}{\rightarrow} \operatorname{det}\left(\frac{\partial^{2} f}{\partial x_{i} \partial x_{j}}\right)
$$

weakly in the sense of measures (note that the expression $\operatorname{det}\left(\frac{\partial^{2} u}{\partial x_{i} \partial x_{j}}\right)$ for a convex function $u$ is understood in a distributional sense, as explained in the quaternionic situation in Theorem 3.4 of $\S 3$ below).

The Chern-Levine-Nirenberg theorem (in fact, in a slightly weaker form) says that if a sequence $\left\{f_{N}\right\}$ of continuous complex plurisubharmonic functions converges uniformly

2000 Mathematics Subject Classification. Primary 31C10, 52A38, 52 A39.

Key words and phrases. HKT-geometry, valuation on convex sets, quaternionic plurisubharmonic functions.

Partially supported by ISF (grant 1369/04). 
on compact subsets to a function $f$, then $f$ is continuous and complex plurisubharmonic, and

$$
\operatorname{det}\left(\frac{\partial^{2} f_{N}}{\partial z_{i} \partial \bar{z}_{j}}\right) \stackrel{w}{\rightarrow} \operatorname{det}\left(\frac{\partial^{2} f}{\partial z_{i} \partial \bar{z}_{j}}\right)
$$

weakly in the sense of measures (again, the expression $\operatorname{det}\left(\frac{\partial^{2} u}{\partial z_{i} \partial \bar{z}_{j}}\right)$ is understood in a distributional sense).

The statement of the quaternionic analog of the above theorems requires analogs of the complex operators $\frac{\partial}{\partial \bar{z}}, \frac{\partial}{\partial z}$ and the notion of the determinant of quaternionic matrices. The former notion is called sometimes the Dirac operators $\frac{\partial}{\partial \bar{q}}, \frac{\partial}{\partial q}$; it is discussed in $\S 2$. The latter notion of quaternionic determinants is described in $\S 1$, where we discuss the Moore determinant of hyper-Hermitian (= quaternionic Hermitian) matrices in detail. This quaternionic result is used in applications to the theory of valuations on convex sets (Theorem 4.2 in $\S 4$ ).

Another result important for applications in valuation theory is Theorem 3.7 (proved in [7]), which is a quaternionic version of Blocki's theorem [13] for complex plurisubharmonic functions.

In $\S 4$ we discuss applications of the above results to the theory of valuations on convex sets in more detail. We recall the basic notions of that theory, referring for further information to the surveys by McMullen [31] and McMullen and Schneider [32]. Let $V$ be a finite-dimensional real vector space. Let $\mathcal{K}(V)$ denote the class of all nonempty compact convex subsets of $V$.

0.1. Definition. (1) A function $\phi: \mathcal{K}(V) \rightarrow \mathbb{C}$ is called a valuation if for any sets $K_{1}, K_{2} \in \mathcal{K}(V)$ such that their union is also convex we have

$$
\phi\left(K_{1} \cup K_{2}\right)=\phi\left(K_{1}\right)+\phi\left(K_{2}\right)-\phi\left(K_{1} \cap K_{2}\right) .
$$

(2) A valuation $\phi$ is said to be continuous if it is continuous with respect to the Hausdorff metric on $\mathcal{K}(V)$.

Recall that the Hausdorff metric $d_{H}$ on $\mathcal{K}(V)$ depends on the choice of a Euclidean metric on $V$ and is defined as follows: $d_{H}(A, B):=\inf \left\{\varepsilon>0 \mid A \subset(B)_{\varepsilon}\right.$ and $\left.B \subset(A)_{\varepsilon}\right\}$, where $(U)_{\varepsilon}$ denotes the $\varepsilon$-neighborhood of a set $U$. Then $\mathcal{K}(V)$ becomes a locally compact space, and the topology on $\mathcal{K}(V)$ induced by the Hausdorff metric does not depend on the choice of a Euclidean metric on $V$.

The theory of valuations has numerous applications in convexity and integral geometry (see, e.g., $[6,23,28,38]$ ). We present some basic examples of translation invariant continuous valuations.

0.2. Example. (1) A Lebesgue measure vol on $V$ is a translation invariant continuous valuation.

(2) The Euler characteristic $\chi$ is a translation invariant continuous valuation. (Recall that $\chi(K)=1$ for any $K \in \mathcal{K}(V)$.)

(3) Let $m:=\operatorname{dim} V$. For a fixed $k=1, \ldots, m$, let $A_{1}, \ldots, A_{m-k} \in \mathcal{K}(V)$. Then the mixed volume

$$
K \mapsto V\left(K[k], A_{1}, \ldots, A_{m-k}\right)
$$

is a translation invariant continuous valuation. (For the notion of the mixed volume and its properties, see, e.g., the books by Burago-Zalgaller [14] and by Schneider [38].)

It was conjectured by McMullen [30] and proved by the author [3] that the linear combinations of the mixed volumes as in Example 0.2 (3) above are dense in the space of all translation invariant continuous valuations in the topology of uniform convergence on the compact subsets of $\mathcal{K}(V)$. 
Nevertheless, there are nontrivial constructions of translation invariant continuous valuations other than mixed volumes. In this survey we shall discuss two of them. They are closely related to each other, being based on the theory of complex and, respectively, quaternionic plurisubharmonic functions. Their relationship with the mixed volume construction is not straightforward. The possibility of approximating these examples by the mixed volumes follows from the solution of McMullen's conjecture.

Let us agree on the notation. For $K \in \mathcal{K}(V)$, we denote by $h_{K}: V^{*} \rightarrow \mathbb{R}$ the supporting functional of $K$. Recall that

$$
h_{K}(y)=\sup \{y(x) \mid y \in K\} .
$$

The construction of valuations via complex plurisubharmonic functions can be described as follows. Let $\Omega^{p, p}$ denote the vector bundle of $(p, p)$-forms over $\mathbb{C}^{n}$, and let $C_{c}\left(\mathbb{C}^{n}, \Omega^{p, p}\right)$ be the space of continuous compactly supported forms of type $(p, p)$ on $\mathbb{C}^{n}$.

0.3. Theorem $\left(\left[7\right.\right.$, Theorem 4.1.3]). Fix $k=1, \ldots, n$. If $\psi \in C_{0}\left(\mathbb{C}^{n}, \Omega^{n-k, n-k}\right)$, then $K \mapsto \int_{\mathbb{C}^{n}}\left(d d^{c} h_{K}\right)^{k} \wedge \psi$ defines a continuous translation invariant valuation on $\mathcal{K}\left(\mathbb{C}^{n}\right)$.

In the above generality, this result was proved by the author in [7]; the proof involves some known properties of complex plurisubharmonic functions. This theorem contains two nontrivial parts: the continuity and the valuation property of the above functional. The former is a consequence of the Chern-Levine-Nirenberg theorem [19], and the latter is a consequence of the Blocki formula [13]. Note that, probably, expressions such as in Theorem 4.1 were considered first in the context of convexity, by Kazarnovskiu in [26] and [27]. In [7] we obtained a quaternionic version of the above construction; it is discussed in $\S 4$, Theorem 4.2 .

In $\S 5$ we discuss two theorems on the Dirichlet problem for quaternionic MongeAmpère equations, obtained by the author in [5]. They also have real and complex classical analogs; see $\S 5$ for references.

In $\S 6$ we describe generalizations of some of the definitions and results on quaternionic plurisubharmonic functions to the so-called hypercomplex manifolds; these generalizations were obtained by Verbitsky and the author in [8]. This class of manifolds contains, for instance, the flat spaces $\mathbb{H}^{n}$ and the hyper-Kähler manifolds. For such manifolds, quaternionic plurisubharmonic functions can be defined, and a version of the Aleksandrov and Chern-Levine-Nirenberg theorems can be proved. Next, it turns out that the $C^{\infty}$-smooth strictly plurisubharmonic functions on hypercomplex manifolds admit a geometric interpretation as (local) potentials of HKT-metrics; this was also shown in [8]. Roughly, an HKT-metric on a hypercomplex manifold is an $S U(2)$-invariant Riemannian metric satisfying certain first order differential equations. These metrics are similar to Kähler metrics on complex manifolds. The above interpretation of quaternionic plurisubharmonic functions is similar to the well-known interpretation of $C^{\infty}$-smooth complex strictly plurisubharmonic functions on complex manifolds as (local) potentials of Kähler metrics. We recall this fact more explicitly. Let $f$ be a $C^{\infty}$-smooth complex strictly plurisubharmonic function on a complex manifold $M$, and let local complex coordinates be fixed on $M$. Then the matrix $g:=\left(\frac{\partial^{2} f}{\partial z_{i} \partial \bar{z}_{j}}\right)$ determines a Kähler metric on $M$. Vice versa, for any Kähler metric $g$ on $M$, every point $z \in M$ has a neighborhood in which $g=\left(\frac{\partial^{2} f}{\partial z_{i} \partial \bar{z}_{j}}\right)$ for a $C^{\infty}$-smooth complex strictly plurisubharmonic function $f$.

The organization of the paper is clear from the table of contents.

\section{§1. Quaternionic Linear ALGEBra}

It is known that some standard results of linear algebra over commutative fields extend to general noncommutative fields, e.g., the theory of the dimension and bases of 
vector spaces. However, over noncommutative fields, no notion of the determinant of matrices is as good as in the commutative case. There is a notion of the Dieudonné determinant (see, e.g., [9]), which is good for some applications (see, e.g., [4]). Gelfand and Retakh have developed a theory of quasideterminants over noncommutative fields which, probably, generalizes in a sense all the known theories of noncommutative determinants (in particular the Dieudonné, the super-, and the quantum determinants). We shall not discuss that theory here, referring the reader to the survey [20].

Nevertheless, over the field $\mathbb{H}$ of quaternions there is a notion of the Moore determinant on the class of hyper-Hermitian matrices discussed below. The hyper-Hermitian quaternionic matrices are similar to real symmetric and complex Hermitian matrices. The properties of the Moore determinant on this class resemble those of the usual determinant. It seems that any general identity or inequality that is true for the determinant of real symmetric or complex Hermitian matrices should be true for the Moore determinants of quaternionic hyper-Hermitian matrices. Among examples of such results, we can mention the Sylvester criterion of positive definiteness and the Aleksandrov inequalities for mixed determinants, discussed below. For more information on quaternionic determinants see the survey [10] and the references therein; for the relationship between the Moore determinant and the Gelfand-Retakh quasideterminants, see [21].

In the remaining part of this section we discuss the notion of hyper-Hermitian matrices and their Moore determinant in more detail.

1.1. Definition. A hyper-Hermitian semilinear form on $V$ is a map $a: V \times V \rightarrow \mathbb{H}$ with the following properties:

(a) $a$ is additive with respect to each argument;

(b) $a(x, y \cdot q)=a(x, y) \cdot q$ for any $x, y \in V$ and any $q \in \mathbb{H}$;

(c) $a(x, y)=\overline{a(y, x)}$, where $\bar{q}$ denotes the usual conjugation of a quaternion $q$.

1.2. Definition. A quaternionic $(n \times n)$-matrix $A=\left(a_{i j}\right)_{i, j=1}^{n}$ is said to be hyperHermitian if $a_{i j}=\bar{a}_{j i}$.

1.3. Example. Let $V=\mathbb{H}^{n}$ be the standard coordinate space viewed as a right vector space over $\mathbb{H}$. Fix a hyper-Hermitian $(n \times n)$-matrix $\left(a_{i j}\right)_{i, j=1}^{n}$. For $x=\left(x_{1}, \ldots, x_{n}\right)$, $y=\left(y_{1}, \ldots, y_{n}\right)$, we define

$$
a(x, y)=\sum_{i, j} \bar{x}_{i} a_{i j} y_{j}
$$

(note the order of the terms!). Then $a$ determines a hyper-Hermitian semilinear form on $V$.

In general, the following standard claims are true.

1.4. Claim. Fix a basis in a finite-dimensional right quaternionic vector space $V$. Then there is a natural bijection between the hyper-Hermitian semilinear forms on $V$ and the $(n \times n)$-hyper-Hermitian matrices.

In fact, this bijection was described in Example 1.3 above.

1.5. Claim. Let $A$ be the matrix of a given hyper-Hermitian form in a given basis. Let $C$ be the transition matrix from this basis to another one. Then the matrix $A^{\prime}$ of the given form in the new basis looks like this: $A^{\prime}=C^{*} A C$.

1.6. Definition. A hyper-Hermitian semilinear form $a$ is positive definite if $a(x, x)>0$ for any nonzero vector $x$.

A quaternionic right vector space $V$ equipped with a fixed positive definite hyperHermitian form $(\cdot, \cdot)$ will be called a hyper-Hermitian space. 
For any quaternionic linear operator $\phi: V \rightarrow V$ in a hyper-Hermitian space $V$, we can define the adjoint operator $\phi^{*}: V \rightarrow V$ in the usual way, i.e., $(\phi x, y)=\left(x, \phi^{*} y\right)$ for any $x, y \in V$. Then, for a fixed orthonormal basis in the space $V$, the operator $\phi$ is selfadjoint if and only if its matrix in this basis is hyper-Hermitian.

1.7. Claim. For any selfadjoint operator $A$ in a hyper-Hermitian space, there exists an orthonormal basis such that the matrix of $A$ in this basis is diagonal and real.

Now we are going to define the Moore determinant of hyper-Hermitian matrices. The definition below differs from that given by Moore in [33], but is equivalent to it.

First, note that every hyper-Hermitian $(n \times n)$ - matrix $A$ determines a hyper-Hermitian semilinear form on the coordinate space $\mathbb{H}^{n}$, as explained in Example 1.3. It also can be viewed as a symmetric bilinear form on $\mathbb{R}^{4 n}$ (which is the realization of $\mathbb{H}^{n}$ ). We denote its $(4 n \times 4 n)$-matrix by ${ }^{\mathbf{R}} A$. The entries of $A$ are regarded as formal variables (each quaternionic entry corresponds to four commuting real variables). Then $\operatorname{det}\left({ }^{\mathbf{R}} A\right)$ is a homogeneous polynomial of degree $4 n$ in $n(2 n-1)$ real variables. Let Id denote the identity matrix. The following result was rediscovered several times (see [10] for references).

1.8. Theorem. There exists a polynomial $P$ defined on the space of all hyper-Hermitian $(n \times n)$-matrices and such that for any hyper-Hermitian $(n \times n)$-matrix $A$ we have $\operatorname{det}\left({ }^{R} A\right)=P^{4}(A)$ and $P(\mathrm{Id})=1$. These two properties determine $P$ uniquely. Furthermore, $P$ is homogeneous of degree $n$ and has integral coefficients.

Thus, for any hyper-Hermitian matrix $A$, the value $P(A)$ is a real number; this number is called the Moore determinant of the matrix $A$. An explicit formula for the Moore determinant was given by Moore [33] (see also [10] and Theorem 1.16 below). In what follows, the Moore determinant of a matrix $A$ will be $\operatorname{denoted}$ by $\operatorname{det} A$. This notation should not cause any confusion with the usual determinant of real or complex matrices, due to part (i) of the next theorem, which is probably folklore.

1.9. Theorem. (i) The Moore determinant of any complex Hermitian matrix viewed as a quaternionic hyper-Hermitian matrix is equal to its usual determinant.

(ii) For any hyper-Hermitian matrix $A$ and any quaternionic matrix $C$ we have

$$
\operatorname{det}\left(C^{*} A C\right)=\operatorname{det} A \cdot \operatorname{det}\left(C^{*} C\right) .
$$

1.10. Example. (a) Let $A=\operatorname{diag}\left(\lambda_{1}, \ldots, \lambda_{n}\right)$ be a diagonal matrix with real $\lambda_{i}$ 's. Then $A$ is hyper-Hermitian and the Moore $\operatorname{determinant} \operatorname{det} A$ is equal to $\prod_{i} \lambda_{i}$.

(b) A general hyper-Hermitian $(2 \times 2)$-matrix $A$ has the form

$$
A=\left[\begin{array}{cc}
a & q \\
\bar{q} & b
\end{array}\right]
$$

where $a, b \in \mathbb{R}$, and $q \in \mathbb{H}$. Then $\operatorname{det} A=a b-q \bar{q}(=a b-\bar{q} q)$.

1.11. Claim. Let $A$ be a nonnegative (respectively, positive) definite hyper-Hermitian matrix. Then $\operatorname{det} A \geq 0$ (respectively, $\operatorname{det} A>0$ ).

The following theorem is a quaternionic generalization of the standard Sylvester criterion.

1.12. Theorem (Sylvester criterion, [4]). A hyper-Hermitian $(n \times n)$ - matrix A is positive definite if and only if the Moore determinants of all the left upper minors of $A$ are positive.

Now we define the mixed discriminant of hyper-Hermitian matrices by analogy with the case of real symmetric matrices studied by A. D. Aleksandrov [1]. 
1.13. Definition. Let $A_{1}, \ldots, A_{n}$ be hyper-Hermitian $(n \times n)$-matrices. Consider the degree $n$ homogeneous polynomial $\operatorname{det}\left(\lambda_{1} A_{1}+\cdots+\lambda_{n} A_{n}\right)$ in real variables $\lambda_{1}, \ldots, \lambda_{n}$. The coefficient of the monomial $\lambda_{1} \cdots \lambda_{n}$ divided by $n$ ! is called the mixed discriminant of the matrices $A_{1}, \ldots, A_{n}$, and is denoted by $\operatorname{det}\left(A_{1}, \ldots, A_{n}\right)$.

Note that the mixed discriminant is symmetric with respect to all variables and linear with respect to each of them; recall that the linearity with respect to, say, the first argument means that

$$
\operatorname{det}\left(\lambda A_{1}^{\prime}+\mu A_{1}^{\prime \prime}, A_{2}, \ldots, A_{n}\right)=\lambda \cdot \operatorname{det}\left(A_{1}^{\prime}, A_{2}, \ldots, A_{n}\right)+\mu \cdot \operatorname{det}\left(A_{1}^{\prime \prime}, A_{2}, \ldots, A_{n}\right)
$$

for any real $\lambda, \mu$. Note also that $\operatorname{det}(A, \ldots, A)=\operatorname{det} A$. The following theorem generalizes the Aleksandrov inequalities for mixed discriminants [1].

1.14. Theorem. (i) The mixed discriminant of positive (respectively, nonnegative) definite matrices is positive (respectively, nonnegative).

(ii) Let $A_{1}, \ldots, A_{n-2}$ be positive definite hyper-Hermitian $(n \times n)$-matrices. On the real linear space of hyper-Hermitian $(n \times n)$-matrices, consider the bilinear form

$$
B(X, Y):=\operatorname{det}\left(X, Y, A_{1}, \ldots, A_{n-2}\right) .
$$

Then $B$ is a nondegenerate quadratic form, and its signature has one plus and the rest are minuses.

1.15. Corollary (Aleksandrov inequality, [4]). Let $A_{1}, \ldots, A_{n-1}$ be positive definite hyper-Hermitian $(n \times n)$-matrices. Then for any hyper-Hermitian matrix $X$ we have

$$
\operatorname{det}\left(A_{1}, \ldots, A_{n-1}, X\right)^{2} \geq \operatorname{det}\left(A_{1}, \ldots, A_{n-1}, A_{n-1}\right) \cdot \operatorname{det}\left(A_{1}, \ldots, A_{n-2}, X, X\right),
$$

and equality occurs if and only if the matrix $X$ is proportional to $A_{n-1}$.

Finally, we present an explicit formula for the Moore determinant (which was the original definition by Moore [33]). Let $A=\left(a_{i, j}\right)_{i, j=1}^{n}$ be a hyper-Hermitian $(n \times n)$ matrix. Let $\sigma$ be a permutation of $\{1, \ldots, n\}$. Write $\sigma$ as a product of disjoint cycles such that each cycle starts with the smallest number. Since disjoint cycles commute, we can write

$$
\sigma=\left(k_{11} \ldots k_{1 j_{1}}\right)\left(k_{21} \ldots k_{2 j_{2}}\right) \ldots\left(k_{m 1} \ldots k_{m j_{m}}\right),
$$

where for each $i$ we have $k_{i 1}<k_{i j}$ for all $j>1$, and $k_{11}>k_{21}>\cdots>k_{m 1}$. This expression is unique. Let $\operatorname{sgn}(\sigma)$ be the parity of $\sigma$. For the next result we refer to [10] and the references therein.

1.16. Theorem. The Moore determinant of $A$ is equal to

$$
\operatorname{det} A=\sum_{\sigma} \operatorname{sgn}(\sigma) a_{k_{11}, k_{12}} \ldots a_{k_{1 j_{1}}, k_{11}} a_{k_{21}, k_{22}} \ldots a_{k_{m j m}, k_{m 1}},
$$

where the sum runs over all permutations $\sigma$.

\section{§2. Dirac Operators}

We write a quaternion $q \in \mathbb{H}$ in the standard form

$$
q=t+x \cdot i+y \cdot j+z \cdot k,
$$

where $t, x, y, z$ are real numbers, and $i, j, k$ satisfy the usual relations

$$
i^{2}=j^{2}=k^{2}=-1, \quad i j=-j i=k, \quad j k=-k j=i, \quad k i=-i k=j .
$$

The Dirac operator $\frac{\partial}{\partial \bar{q}}$ is defined as follows. For any $\mathbb{H}$-valued function $F$,

$$
\frac{\partial}{\partial \bar{q}} F:=\frac{\partial F}{\partial t}+i \frac{\partial F}{\partial x}+j \frac{\partial F}{\partial y}+k \frac{\partial F}{\partial z} .
$$


Also, we define the operator $\frac{\partial}{\partial q}$ :

$$
\frac{\partial}{\partial q} F:=\overline{\frac{\partial}{\partial q} \bar{F}}=\frac{\partial F}{\partial t}-\frac{\partial F}{\partial x} i-\frac{\partial F}{\partial y} j-\frac{\partial F}{\partial z} k .
$$

In the case of several quaternionic variables, it is easily seen that the operators $\frac{\partial}{\partial q_{i}}$ and $\frac{\partial}{\partial \bar{q}_{j}}$ commute:

$$
\left[\frac{\partial}{\partial q_{i}}, \frac{\partial}{\partial \bar{q}_{j}}\right]=0
$$

2.1. Proposition ([4]). (i) Let $f: \mathbb{H}^{n} \rightarrow \mathbb{H}$ be a smooth function. Then for any $\mathbb{H}-$ linear transformation $A$ of the space $\mathbb{H}^{n}$ (viewed as a right $\mathbb{H}$-vector space) we have the identities

$$
\left(\frac{\partial^{2} f(A q)}{\partial \bar{q}_{i} \partial q_{j}}\right)=A^{*}\left(\frac{\partial^{2} f}{\partial \bar{q}_{i} \partial q_{j}}(A q)\right) A .
$$

(ii) If, moreover, $f$ is real-valued, then for any $\mathbb{H}$-linear transformation $A$ of $\mathbb{H}^{n}$ and any quaternion a with $|a|=1$ we have

$$
\left(\frac{\partial^{2} f(A(q \cdot a))}{\partial \bar{q}_{i} \partial q_{j}}\right)=A^{*}\left(\frac{\partial^{2} f}{\partial \bar{q}_{i} \partial q_{j}}(A(q \cdot a))\right) A .
$$

\section{§3. Plurisubharmonic Functions of Quaternionic Variables}

First, we follow [4] to introduce the class of quaternionic plurisubharmonic functions on $\mathbb{H}^{n}$. The same notion was also introduced independently by Henkin in [24]. Let $\Omega$ be an open subset of $\mathbb{H}^{n}$.

3.1. Definition. A real-valued function $u: \Omega \rightarrow \mathbb{R}$ is said to be quaternionic plurisubharmonic if it is upper semicontinuous and its restriction to any right quaternionic line is subharmonic.

Recall that upper semicontinuity means that $u\left(x_{0}\right) \geq \lim \sup _{x \rightarrow x_{0}} u(x)$ for any $x_{0} \in \Omega$. The class of plurisubharmonic functions on $\Omega$ will be denoted by $P(\Omega)$.

Also, we say that a $C^{2}$-smooth function $u: \Omega \rightarrow \mathbb{R}$ is strictly plurisubharmonic if its restriction to any right quaternionic line is strictly subharmonic (i.e., the Laplacian is strictly positive).

Before stating the next proposition, we observe that if a smooth function $f$ is realvalued, then the matrix $\left(\frac{\partial^{2} f}{\partial q_{i} \partial \bar{q}_{j}}\right)(q)$ is hyper-Hermitian.

3.2. Proposition ([4, Proposition 2.1.6]). A real-valued and twice continuously differentiable function $f$ on an open subset $\Omega \subset \mathbb{H}^{n}$ is quaternionic plurisubharmonic (respectively, strictly plurisubharmonic) if and only if at every point $q \in \Omega$ the matrix $\left(\frac{\partial^{2} f}{\partial q_{i} \partial \bar{q}_{j}}\right)(q)$ is nonnegative definite (respectively, positive definite).

3.3. Remark. Proposition 3.2 is completely similar to the characterization of the smooth convex functions as those for which the Hessian $\left(\frac{\partial^{2} f}{\partial x_{i} \partial x_{j}}\right)$ is nonnegative definite, and the characterization of the smooth complex plurisubharmonic functions as those for which the complex Hessian $\left(\frac{\partial^{2} f}{\partial z_{i} \partial \bar{z}_{j}}\right)$ is nonnegative definite.

3.4. Theorem $([4])$. For any $u \in C(\Omega) \cap P(\Omega)$, a nonnegative measure $\operatorname{det}\left(\frac{\partial^{2} u}{\partial q_{i} \partial \bar{q}_{j}}\right)$ can be defined uniquely; this measure is characterized by the following two properties:

(1) if $u \in C^{2}(\Omega)$, then the above measure has an obvious meaning; 
(2) if $u_{N} \rightarrow u$ uniformly on the compact subsets of $\Omega$, and $u_{N}, u \in C(\Omega) \cap P(\Omega)$, then

$$
\operatorname{det}\left(\frac{\partial^{2} u_{N}}{\partial q_{i} \partial \bar{q}_{j}}\right) \stackrel{w}{\rightarrow} \operatorname{det}\left(\frac{\partial^{2} u}{\partial q_{i} \partial \bar{q}_{j}}\right),
$$

where the convergence of measures is understood in the weak sense.

3.5. Remark. (1) It is easily seen that if $u_{N} \rightarrow u$ uniformly on compact subsets and $u_{N} \in C(\Omega) \cap P(\Omega)$, then $u \in C(\Omega) \cap P(\Omega)$.

(2) Note that the real analog of this result was proved by Aleksandrov in [2], and the complex analogue was obtained by Chern, Levine, and Nirenberg in [19].

We shall need a refinement of Theorem 3.4, which was proved by the author in [7] in somewhat different notation. We denote $\partial^{2} u:=\left(\frac{\partial^{2} u}{\partial q_{i} \partial \bar{q}_{j}}\right)$ for brevity.

3.6. Theorem (see [7]). Let $\Omega \subset \mathbb{H}^{n}$ be an open subset. Fix $k=1, \ldots, n$. Let $\left\{u_{N}^{(i)}\right\}_{N=1}^{\infty}$, $1 \leq i \leq k$, be sequences in $P(\Omega) \cap C(\Omega)$, and let $V^{(1)}, \ldots, V^{(n-k)}$ be continuous functions on $\Omega$ with values in the space of $(n \times n)$-hyper-Hermitian matrices. Assume that, for every $i=1, \ldots, k$,

$$
u_{N}^{(i)} \rightarrow u^{(i)} \text { as } N \rightarrow \infty
$$

uniformly on compact subsets. Then $u^{(i)} \in P(\Omega) \cap C(\Omega)$, and

$$
\operatorname{det}\left(\partial^{2} u_{N}^{(1)}, \ldots, \partial^{2} u_{N}^{(k)}, V^{(1)}, \ldots, V^{(n-k)}\right) \stackrel{w}{\rightarrow} \operatorname{det}\left(\partial^{2} u^{(1)}, \ldots, \partial^{2} u^{(k)}, V^{(1)}, \ldots, V^{(n-k)}\right)
$$

weakly in the sense of measures (we have used the notion of the mixed determinant).

For hyper-Hermitian matrices $A, B_{1}, \ldots, B_{n-k}$, we denote

$$
\operatorname{det}\left(A[k], B_{1}, \ldots, B_{n-k}\right):=\operatorname{det}(\underbrace{A, \ldots, A}_{k \text { times }}, B_{1}, \ldots, B_{n-k}) .
$$

Observe that the maximum of two plurisubharmonic functions is plurisubharmonic.

3.7. Theorem (see [7]). Let $\Omega \subset \mathbb{H}^{n}$ be an open subset. Fix $k=1, \ldots, n$. Let $f, g \in$ $P(\Omega) \cap C(\Omega)$. Assume that $\min \{f, g\} \in P(\Omega) \cap C(\Omega)$. If $V^{(1)}, \ldots, V^{(n-k)}$ are continuous functions on $\Omega$ with values in the space of $(n \times n)$-hyper-Hermitian matrices, then

$$
\begin{aligned}
\operatorname{det}\left(\partial^{2}(\max \{f, g\})[k], V^{(1)}, \ldots, V^{(n-k)}\right) & \\
= & \operatorname{det}\left(\partial^{2} f[k], V^{(1)}, \ldots, V^{(n-k)}\right)+\operatorname{det}\left(\partial^{2} g[k], V^{(1)}, \ldots, V^{(n-k)}\right) \\
& -\operatorname{det}\left(\partial^{2}(\min \{f, g\})[k], V^{(1)}, \ldots, V^{(n-k)}\right) .
\end{aligned}
$$

3.8. Remark. Theorem 3.7 was proved in [7] as a consequence of a more precise result, Theorem 3.2.1 there, which is a quaternionic version of a result proved by Blocki in [13] for complex plurisubharmonic functions.

\section{§4. Applications to valuation theory}

Here we discuss applications of the above results to the theory of valuations on convex sets. The necessary definitions of this theory were recalled in the Introduction. Especially, we recall Theorem 0.3 .

4.1. Theorem ([7, Theorem 4.1.3]). Fix $k=1, \ldots, n$ and $\psi \in C_{0}\left(\mathbb{C}^{n}, \Omega^{n-k, n-k}\right)$. Then the rule $K \mapsto \int_{\mathbb{C}^{n}}\left(d d^{c} h_{K}\right)^{k} \wedge \psi$ determines a continuous translation invariant valuation on $\mathcal{K}\left(\mathbb{C}^{n}\right)$. 
In the above generality, this result was proved by the author in [7]; the proof involves some known properties of complex plurisubharmonic functions. This theorem contains two nontrivial parts: the continuity and the valuation property of the above functional. Continuity is a consequence of the Chern-Levine-Nirenberg theorem [19] and the fact that a sequence $\left\{K_{N}\right\}$ of convex compact sets converges in the Hausdorff metric to a convex compact set $K$ if and only if $h_{K_{N}} \rightarrow h_{K}$ uniformly on compact subsets. The valuation property is a consequence of the Blocki formula (see [13]) combined with the fact that

$$
\begin{aligned}
& h_{K_{1} \cup K_{2}}=\max \left\{h_{K_{1}}, h_{K_{2}}\right\} \text { whenever } K_{1} \cup K_{2} \text { is convex, and } \\
& h_{K_{1} \cap K_{2}}=\min \left\{h_{K_{1}}, h_{K_{2}}\right\} .
\end{aligned}
$$

Let us discuss the quaternionic version of Theorem 4.1. To facilitate the exposition, we state the result in a more straightforward form. A better way was discussed in [7], where quaternionic analogs of $(p, p)$-forms were introduced.

4.2. Theorem ([7, Theorem 4.2.1]). Fix $k=1, \ldots, n$. Let $\psi_{0}$ be a continuous compactly supported real-valued function on $\mathbb{H}^{n *}$, and let $V^{(1)}, \ldots, V^{(n-k)}$ be continuous compactly supported functions on $\mathbb{H}^{n *}$ with values in the space of $(n \times n)$-hyper-Hermitian matrices. Then the functional

$$
K \mapsto \int_{\mathbb{H}^{n *}} \operatorname{det}\left(\partial^{2} h_{K}[k], V^{(1)}, \ldots, V^{(n-k)}\right) \cdot \psi_{0} \cdot d \text { vol }
$$

is a translation invariant continuous valuation.

As in the complex case, continuity is a consequence of Theorem 3.6, and the valuation property is a consequence of Theorem 3.7 .

\section{§5. Quaternionic Monge-Ampère equations}

In this section we discuss some results on quaternionic Monge-Ampère equations; we follow [5]. These results have real and complex analogs; the corresponding references will be given below.

5.1. Definition. An open bounded domain $\Omega \subset \mathbb{H}^{n}$ with a smooth boundary $\partial \Omega$ is said to be strictly pseudoconvex if for every point $z_{0} \in \partial \Omega$ there exists a neighborhood $\mathcal{O}$ and a smooth quaternionic strictly plurisubharmonic function $h$ on $\mathcal{O}$ such that $\Omega \cap \mathcal{O}=$ $\{h<0\}, h\left(z_{0}\right)=0$, and $\nabla h\left(z_{0}\right) \neq 0$.

Let $B$ denote the unit Euclidean ball in $\mathbb{H}^{n}$.

5.2. Theorem ([5, Theorem 0.1.4]). Let $f \in C^{\infty}(\bar{B}), f>0$. For any $\phi \in C^{\infty}(\partial B)$ there exists a unique function $u \in C^{\infty}(\bar{B})$ that is quaternionic plurisubharmonic in $B$ and solves the Dirichlet problem

$$
\operatorname{det}\left(\frac{\partial^{2} u}{\partial q_{i} \partial \bar{q}_{j}}\right)=f \text { in } B,\left.\quad u\right|_{\partial B}=\phi .
$$

5.3. Remark. (1) It is natural to expect that Theorem 5.2 is true for a larger class of domains, say for all bounded strictly pseudoconvex domains.

(2) The real version of Theorem 5.2 was proved for arbitrary bounded strictly convex domains in $\mathbb{R}^{n}$ by Caffarelli, Nirenberg, and Spruck [15]. The complex version was proved for arbitrary bounded strictly pseudoconvex domains in $\mathbb{C}^{n}$ by Caffarelli, Kohn, Nirenberg, and Spruck [16], and Krylov [29]. Our method is a modification of the method used in [16]. We also note that for $n=1$ the problem reduces to the classical Dirichlet problem for the Laplacian in $\mathbb{R}^{4}$ (which is a linear problem); it was solved in the XIX 
century. The interior regularity of the solution of the Dirichlet problem for real MongeAmpère equations had been proved before by A. Pogorelov, and the proof was described briefly in [34]-[36]. The complete proof was published in [37] and [17, 18].

5.4. Theorem ([5, Theorem 0.1.3]). Let $\Omega \subset \mathbb{H}^{n}$ be a bounded quaternionic strictly pseudoconvex domain. Let $f \in C(\bar{\Omega}), f \geq 0$. For any $\phi \in C(\partial \Omega)$ there exists a unique function $u \in C(\bar{\Omega})$ plurisubharmonic in $\Omega$ and such that

$$
\operatorname{det}\left(\frac{\partial^{2} u}{\partial q_{i} \partial \bar{q}_{j}}\right)=f \text { in } \Omega,\left.\quad u\right|_{\partial \Omega} \equiv \phi .
$$

5.5. Remark. The real analogue of this result was proved by Aleksandrov in [2], and the complex one by Bedford and Taylor in [12].

\section{§6. Generalizations to hypercomplex manifolds}

Some of the definitions and results on quaternionic plurisubharmonic functions discussed above were extended by Verbitsky and the author [8] to a more general context of the so-called hypercomplex manifolds. In this section we give an overview of these results, including a geometric interpretation of the quaternionic strictly plurisubharmonic functions as (local) potentials of HKT-metrics. The exposition follows [8].

6.1. Definition. A hypercomplex manifold is a smooth manifold $X$ together with a triple $(I, J, K)$ of complex structures satisfying the usual quaternionic relations:

$$
I J=-J I=K \text {. }
$$

6.2. Remark. (1) Here we suppose (contrary to much of the literature on the subject) that the complex structures $I, J, K$ act from the right on the tangent bundle $T X$ of $X$. This action extends uniquely to a right action of the algebra $\mathbb{H}$ of quaternions on $T X$.

(2) It follows that the dimension of a hypercomplex manifold $X$ is divisible by 4 .

Let $\left(X^{4 n}, I, J, K\right)$ be a hypercomplex manifold. We denote by $\Lambda_{I}^{p, q}(X)$ the vector bundle of $(p, q)$-forms on the complex manifold $(X, I)$. By abuse of notation, we shall also denote the space of $C^{\infty}$-sections of this bundle by the same symbol $\Lambda_{I}^{p, q}(X)$.

Let

$$
\partial: \Lambda_{I}^{p, q}(X) \rightarrow \Lambda_{I}^{p+1, q}(X)
$$

be the usual $\partial$-differential of differential forms on the complex manifold $(X, I)$.

Set

$$
\partial_{J}:=J^{-1} \circ \bar{\partial} \circ J
$$

6.3. Claim ([39]). (1) $J: \Lambda_{I}^{p, q}(X) \rightarrow \Lambda_{I}^{q, p}(X)$.

(2) $\partial_{J}: \Lambda_{I}^{p, q}(X) \rightarrow \Lambda_{I}^{p+1, q}(X)$.

(3) $\partial \partial_{J}=-\partial_{J} \partial$.

6.4. Definition ([39]). Let $k=0,1, \ldots, n$. A form $\omega \in \Lambda_{I}^{2 k, 0}(X)$ is said to be real if

$$
\overline{J \circ \omega}=\omega .
$$

The subspace of real $C^{\infty}$-smooth $(2 k, 0)$-forms on $(X, I)$ will be denoted by $\Lambda_{I, \mathbb{R}}^{2 k, 0}(X)$.

6.5. Lemma. Let $X$ be a hypercomplex manifold. Let $f: X \rightarrow \mathbb{R}$ be a smooth function. Then $\partial \partial_{J} f \in \Lambda_{I, \mathbb{R}}^{2,0}(X)$. 
6.6. Definition. Let $\omega \in \Lambda_{I, \mathbb{R}}^{2,0}(X)$. We say that $\omega$ is nonnegative (notation: $\omega \geq 0$ ) if

$$
\omega(Y, Y \circ J) \geq 0
$$

for any (real) vector field $Y$ on the manifold $X$. Equivalently, $\omega$ is nonnegative if $\omega(Z, \bar{Z} \circ J) \geq 0$ for any $(1,0)$-vector field $Z$.

6.7. Definition. A continuous function

$$
h: X \rightarrow \mathbb{R}
$$

is said to be quaternionic plurisubharmonic if $\partial \partial_{J} h$ is a nonnegative (generalized) section of $\Lambda_{I, \mathbb{R}}^{2,0}(X)$.

6.8. Remark. The generalized nonnegativity was discussed in detail in $[8, \S 5]$.

Let $P^{\prime}(X)$ denote the class of continuous quaternionic plurisubharmonic functions on $X$, and let $P^{\prime \prime}(X)$ be the subclass of functions in $P^{\prime}(X)$ with the following additional property: a function $h \in P^{\prime}(X)$ belongs to $P^{\prime \prime}(X)$ if and only if for any point $x \in X$ there is a neighborhood $U \ni x$ and a sequence $\left\{h_{N}\right\} \subset P^{\prime}(U) \cap C^{2}(U)$ such that $h_{N} \rightarrow h$ uniformly on the compact subsets of $U$. Thus, $P^{\prime \prime}(X) \subset P^{\prime}(X)$.

We conjecture that $P^{\prime}(X)=P^{\prime \prime}(X)$. This conjecture is true in the case where $X$ is an open subset of $\mathbb{H}^{n}$.

6.9. Theorem ([8, Theorem 1.10]). Let $X$ be a hypercomplex manifold of (real) dimension $4 n$. Let $0<k \leq n$. For any $h^{(1)}, \ldots, h^{(k)} \in P^{\prime \prime}(X)$, a nonnegative generalized section of $\Lambda_{I, \mathbb{R}}^{2 k}$ denoted by $\partial \partial_{J} h^{(1)} \wedge \cdots \wedge \partial \partial_{J} h^{(k)}$ can be defined; this section is characterized uniquely by the following two properties:

(1) if $h^{(1)}, \ldots, h^{(k)} \in C^{2}(X)$, then the definition is clear;

(2) if $\left\{h_{N}^{(i)}\right\} \subset P^{\prime \prime}(X)$ and $h_{N}^{(i)} \rightarrow h^{(i)}$ as $N \rightarrow \infty$ uniformly on compact subsets, $i=1$, $\ldots, k$, then $h^{(i)} \in P^{\prime \prime}(X)$ and

$$
\partial \partial_{J} h_{N}^{(1)} \wedge \cdots \wedge \partial \partial_{J} h_{N}^{(k)} \rightarrow \partial \partial_{J} h^{(1)} \wedge \cdots \wedge \partial \partial_{J} h^{(k)}
$$

in the weak topology on measures.

6.10. Remark. Theorem 6.9 extends Theorem 3.6 from the flat space $\mathbb{H}^{n}$ to the hypercomplex manifolds.

We discuss the links to the HKT-geometry. Let $g$ be a Riemannian metric on a hypercomplex manifold $X$. The metric $g$ is said to be quaternionic Hermitian (or hyperHermitian) if $g$ is invariant with respect to the group $S U(2) \subset \mathbb{H}$ of unitary quaternions.

Given a quaternionic Hermitian metric $g$ on a hypercomplex manifold $X$, consider the differential form

$$
\Omega:=\omega_{J}-\sqrt{-1} \omega_{K},
$$

where $\omega_{L}(A, B):=g(A, B \circ L)$ for any $L \in \mathbb{H}$ with $L^{2}=-1$ and any real vector fields $A, B$ on $X$. It is easily seen that $\Omega$ is a $(2,0)$-form with respect to the complex structure $I$.

6.11. Definition. The metric $g$ on $X$ is called an HKT-metric if $\partial \Omega=0$.

6.12. Remark. The HKT-metrics on hypercomplex manifolds first were introduced by Howe and Papadopoulos in [25]. Their original definition was different from but equivalent to Definition 6.11 (see [22]).

We denote by $S_{\mathbb{H}}(X)$ the vector bundle over $X$ such that its fiber over a point $x \in X$ is the space of hyper-Hermitian forms on the tangent space $T_{x} X$. Consider the map of vector bundles

$$
t: \Lambda_{I, \mathbb{R}}^{2,0}(X) \rightarrow S_{\mathbb{H}}(X)
$$


defined by $t(\eta)(A, A)=\eta(A, A \circ J)$ for any (real) vector field $A$ on $X$. Then $t$ is an isomorphism of vector bundles (this was proved in [39]).

6.13. Theorem ([8, Proposition 1.14]). (1) Let $f$ be an infinitely smooth strictly plurisubharmonic function on a hypercomplex manifold $(X, I, J, K)$. Then $t\left(\partial \partial_{J} f\right)$ is an HKTmetric.

(2) Conversely, assume that $g$ is an HKT-metric. Then for any point $x \in X$ there is a neighborhood $U$ of $x$ and an infinitely smooth strictly plurisubharmonic function $f$ on $U$ such that $g=t\left(\partial \partial_{J} f\right)$ in $U$.

6.14. Remark. (i) By [8, Proposition 4.1], on the flat space $\mathbb{H}^{n}$, for any smooth realvalued function $f$ we have

$$
t\left(\partial \partial_{J} f\right)=\frac{1}{4}\left(\frac{\partial^{2} f}{\partial q_{i} \partial \bar{q}_{j}}\right) .
$$

(ii) The proof of Theorem 6.13 employs a result of Banos-Swann [11].

\section{ACKNOWLEDGEMENTS}

I thank G. Henkin and M. Verbitsky for very useful discussions.

\section{REFERENCES}

[1] A. D. Aleksandrov, Zur Theorie der gemischten Volumina von konvexen Körpern. IV. Die gemischten Diskriminanten und die gemischten Volumina, Mat. Sb. (N.S.) 3 (45) (1938), no. 2, $227-251$. (Russian) (German summary).

[2] Dirichlet's problem for the equation Det $\left\|z_{i j}\right\|=\varphi\left(z_{1}, \ldots, z_{n}, z, x_{1}, \ldots, x_{n}\right)$. I, Vestnik Leningrad. Univ. Ser. Mat. Mekh. Astronom. 1958, vyp. 1, 5-24. (Russian) MR0096903 (20:3385)

[3] S. Alesker, Description of translation invariant valuations on convex sets with solution of P. McMullen's conjecture, Geom. Funct. Anal. 11 (2001), no. 2, 244-272. MR1837364 (2002e:52015)

[4] Non-commutative linear algebra and plurisubharmonic functions of quaternionic variables, Bull. Sci. Math. 127 (2003), no. 1, 1-35; also: math.CV/0104209. MR1957796 (2004b:32054)

[5] _ Quaternionic Monge-Ampère equations, J. Geom. Anal. 13 (2003), no. 2, 205-238; also: math.CV/0208005. MR1967025 (2004d:32055)

[6] _ Hard Lefschetz theorem for valuations, complex integral geometry, and unitarily invariant valuations, J. Differential Geom. 63 (2003), 63-95; also: math.MG/0209263. MR2015260 (2004h:52015)

[7] - Valuations on convex sets, non-commutative determinants, and pluripotential theory, Adv. Math. 195 (2005), no. 2, 561-595; also: math.MG/0401219. MR2146354 (2006f:32047)

[8] S. Alesker and M. Verbitsky, Plurisubharmonic functions on hypercomplex manifolds and HKTgeometry, J. Geom. Anal. 16 (2006), no. 3, 375-399; also: math.CV/0510140. MR2250051 (2007e:32042)

[9] E. Artin, Geometric algebra, Intersci. Publ., Inc., New York-London, 1957. MR0082463 (18:553e)

[10] H. Aslaksen, Quaternionic determinants, Math. Intelligencer 18 (1996), no. 3, 57-65. MR1412993 (97j:16028)

[11] B. Banos and A. Swann, Potentials for hyper-Kähler metrics with torsion, Classical Quantum Gravity 21 (2004), no. 13, 3127-3135. MR2072130 (2005c:53054)

[12] E. Bedford and B. A. Taylor, The Dirichlet problem for a complex Monge-Ampère equation, Invent. Math. 37 (1976), no. 1, 1-44. MR0445006 (56:3351)

[13] Z. Błocki, Equilibrium measure of a product subset of $C^{n}$, Proc. Amer. Math. Soc. 128 (2000), no. 12, 3595-3599. MR1707508 (2001b:32072)

[14] Yu. D. Burago and V. A. Zalgaller, Geometric inequalities, "Nauka", Leningrad, 1980; English transl., Grundlehren Math. Wiss., Bd. 285, Springer-Verlag, Berlin, 1988. MR0602952 (82d:52009); MR0936419 (89b:52020)

[15] L. Caffarelli, L. Nirenberg, and J. Spruck, The Dirichlet problem for nonlinear second-order elliptic equations. I. Monge-Ampère equation, Comm. Pure Appl. Math. 37 (1984), no. 3, 369-402. MR0739925 (87f:35096)

[16] L. Caffarelli, J. J. Kohn, L. Nirenberg, and J. Spruck, The Dirichlet problem for nonlinear secondorder elliptic equations. II. Complex Monge-Ampère, and uniformly elliptic, equations, Comm. Pure Appl. Math. 38 (1985), no. 2, 209-252. MR0780073 (87f:35097) 
[17] Shiu Yuen Cheng and Shing Tung Yau, On the regularity of the Monge-Ampère equation $\operatorname{det}\left(\partial^{2} u / \partial x_{i} \partial x_{j}\right)=F(x, u)$, Comm. Pure Appl. Math. 30 (1977), no. 1, 41-68. MR0437805 (55:10727)

[18] _ The real Monge-Ampère equation and affine flat structures, Proceedings of the 1980 Beijing Symposium on Differential Geometry and Differential Equations, Vols. 1, 2, 3 (Beijing, 1980), Science Press, Beijing, 1982, pp. 339-370. MR0714338 (85c:53103)

[19] S. S. Chern, H. I. Levine, and L. Nirenberg, Intrinsic norms on a complex manifold, 1969 Global Analysis (Papers in Honor of K. Kodaira), Univ. Tokyo Press, Tokyo, 1969, pp. 119-139. MR0254877 (40:8084)

[20] I. Gelfand, S. Gelfand, V. Retakh, and R. Lee Wilson, Quasideterminants, Adv. Math. 193 (2005), no. 1, 56-141. MR2132761 (2006a:05165)

[21] I. Gelfand, V. Retakh, and R. Lee Wilson, Quaternionic quasideterminants and determinants, Lie Groups and Symmetric Spaces, Amer. Math. Soc. Transl. Ser. 2, vol. 210, Amer. Math. Soc., Providence, RI, 2003, pp. 111-123; also: math.QA/0206211. MR2018356 (2004j:15010)

[22] G. Grantcharov and Y. S. Poon, Geometry of hyper-Kähler connections with torsion, Comm. Math. Phys. 213 (2000), no. 1, 19-37. MR1782143 (2002a:53059)

[23] H. Hadwiger, Vorlesungen über Inhalt, Oberfläche und Isoperimetrie, Springer-Verlag, Berlin, 1957. MR0102775 (21:1561)

[24] G. Henkin, Private communication.

[25] P. S. Howe and G. Papadopoulos, Twistor spaces for hyper-Kähler manifolds with torsion, Phys. Lett. B 379 (1996), no. 1-4, 80-86. MR1396267 (97h:53073)

[26] B. Ya. Kazarnovskiǔ, On zeros of exponential sums, Dokl. Akad. Nauk SSSR 257 (1981), no. 4, 804-808; English transl. in Soviet Math. Dokl. 23 (1981). MR0612571 (82i:32014)

[27] Newton polyhedra and roots of systems of exponential sums, Funktsional. Anal. i Prilozhen. 18 (1984), no. 4, 40-49; English transl., Funct. Anal. Appl. 18 (1984), no. 4, 299-307. MR0775932 (87b:32005)

[28] D. Klain and G.-C. Rota, Introduction to geometric probability, Lincei Lectures, Cambridge Univ. Press, Cambridge, 1997. MR1608265 (2001f:52009)

[29] N. V. Krylov, Smoothness of the payoff function for a controllable diffusion process in a domain, Izv. Akad. Nauk SSSR Ser. Mat. 53 (1989), no. 1, 66-96; English transl., Math. USSR-Iz. 34 (1990), no. 1, 65-95. MR0992979 (90f:93040)

[30] P. McMullen, Continuous translation-invariant valuations on the space of compact convex sets, Arch. Math. (Basel) 34 (1980), no. 4, 377-384. MR0593954 (81m:52013)

[31] _ Valuations and dissections, Handbook of Convex Geometry, Vols. A, B, North-Holland, Amsterdam, 1993, pp. 933-988. MR1243000 (95f:52018)

[32] P. McMullen and R. Schneider, Valuations on convex bodies, Convexity and its Applications, Birkhäuser, Basel, 1983, pp. 170-247. MR0731112 (85e:52001)

[33] E. H. Moore, On the determinant of an hermitian matrix of quaternionic elements, Bull. Amer. Math. Soc. 28 (1922), 161-162.

[34] A. V. Pogorelov, The regularity of the generalized solutions of the equation $\operatorname{det}\left(\partial^{2} u / \partial x^{i} \partial x^{j}\right)=$ $\varphi\left(x^{1}, x^{2}, \ldots, x^{n}\right)>0$, Dokl. Akad. Nauk SSSR 200 (1971), no. 3, 534-537; English transl., Soviet Math. Dokl. 12 (1971), 1436-1440. MR0293227 (45:2304)

[35] - The Dirichlet problem for the multidimensional analogue of the Monge-Ampère equation, Dokl. Akad. Nauk SSSR 201 (1971), no. 4, 790-793; English transl., Soviet Math. Dokl. 12 (1971), 1227-1231. MR0293228 (45:2305)

[36] - A regular solution of the $n$-dimensional Minkowski problem, Dokl. Akad. Nauk SSSR 199 (1971), no. 4, 785-788; English transl., Soviet Math. Dokl. 12 (1971), 1192-1196. MR0284956 $(44: 2180)$

[37] — The multidimensional Monge-Ampère equation $\operatorname{det}\left\|z_{i j}\right\|=\phi\left(z_{1}, \ldots, z_{n}, z, x_{1}, \ldots, x_{n}\right)$, "Nauka", Moscow, 1988. (Russian) MR0938951 (89g:35036)

[38] R. Schneider, Convex bodies: the Brunn-Minkowski theory, Encyclopedia Math. Appl., vol. 44, Cambridge Univ. Press, Cambridge, 1993. MR1216521 (94d:52007)

[39] M. Verbitsky, Hyper-Kähler manifolds with torsion, supersymmetry and Hodge theory, Asian J. Math. 6 (2002), no. 4, 679-712. MR1958088 (2004e:53071)

Department of Mathematics, Tel Aviv University, Ramat Aviv, 69978 Tel Aviv, Israel

E-mail address: semyon@post.tau.ac.il

Received 1/AUG/2006

Originally published in English 